\title{
Cognitive Benefits of Being Bilingual for Young Children: A Literature Review
}

\author{
Weishuang Guo ${ }^{1}$, Shushi $\mathrm{Yao}^{2, *}$ \\ ${ }^{1}$ University of British Columbia, Vancouver, Canada \\ ${ }^{2}$ Teachers College, Columbia University, New York, United States \\ ${ }^{*}$ Corresponding author. Email: sy2927@tc.columbia.edu \\ These authors contributed equally.
}

\begin{abstract}
Being bilingual is prevalent and is on the rise worldwide. Although there were some people discussing the null result of being bilingual in cognitive aspects (executive functions, metacognition), there were lots of investigations demonstrating that being bilingual exhibited many aspects of benefits in cognitive development for young children. Frequently, articles and investigations paid attention to one benefit or phenomenon in this area. This review article aims to summarize and examine three main bilingual cognitive benefits in executive functions (inhibitory control, working memory, shifting), enhanced language skills, and neurological changes for young children. In conclusion, we find that being bilingual leads to positive and beneficial results than null results.
\end{abstract}

Keywords: Bilingual, Young children, Cognitive Benefit

\section{INTRODUCTION}

Bilingualism is common and is on the rise in many parts of the world. For example, according to the 2016 European Commission survey, more than $80 \%$ of European Union citizens speak at least one foreign language [1]. From the 2017 Statistics Canada report, $54.5 \%$ of people with a native language other than English or French used their native language and an official language at home [2]. Even in the United States, which is widely considered to be monolingual, $21.9 \%$ of U.S. residents spoke a foreign language at home in 2018, more than double the 11 percent in 1980 [3]. Although no worldwide data existed, it has been estimated that about half of the world's population is bilingual [4]. Such widespread bilingualism motivated researchers to investigate the effect of bilingualism on cognitive development.

This article will discuss the potential cognitive benefits associated with being bilingual for young children from three aspects. Firstly, we will review the effect of being bilingual on executive functions, including inhibitory control, working memory, and shifting. Secondly, we will discuss how language abilities are changed due to acquiring a second language. Thirdly, to investigate the underlying mechanism for these cognitive benefits of being bilingual, evidence of the brain's structural changes in the bilingual populations will be discussed.

Regarding executive functions, Santillán \& Khurana examined a study to suggest that bilinguals were associated with quicker inhibitory control by comparing with three groups with different language experiences [5]. Additionally, Hilchey \& Klein discovered that bilinguals have better inhibitory control on both compatible and incompatible tests [6]. Filippi et al. inspected that bilinguals are more likely to ignore distractors to understand complex sentences than monolinguals [7]. Garcia et al. explored that bilinguals had enhanced working memory which had a positive effect on children's mathematical development [8]. Daubert \& Ramani furthermore maintained that being bilingual for children with disruptive behavior disorders displayed fewer working memory problems [9]. Mehrani \& Zabihi concluded that young bilingual children show higher scores on shifting tasks and Prior $\&$ Macwhinney also argued being bilingual had less shifting costs by doing shifting tests $[10,11]$.

Concerning language abilities, young bilinguals have the better capability of acquiring a third language compared with monolinguals due to their enhanced metalinguistic awareness[12]. In other words, young bilinguals are more likely to comprehend arbitrary word 
meanings and forms. Moreover, Weikum et al. also insisted that young bilinguals show better development of visual language discrimination by showing infants a slient video clip with two languages [13].

With respect to neurological changes, Klein et al. contended that bilingual young children had increased thickness of the left inferior frontal cortex, which was a significant area in the brain for speech development [14]. Moreover, Elmer et al. and Piliasikas et al. stated that children who are bilingual also had a structural change of white matter, which leads to an enhancement in children's speech and semantic development.

However, other researchers find inconsistent results regarding the cognitive benefits of being bilinguals. For example, Nichols et al. assessed general executive functions between monolinguals and bilinguals and found no reliable differences between groups [15]. In addition, Duñabeitia et al. and Antón et al. tested inhibitory skills and came to the same conclusion that the cognitive function of bilinguals is not better than that of monolinguals $[16,17]$. Further, Folke et al. found a bilingual disadvantage in metacognition by examining confidence judgments of perceptual tasks [18].

How bilingual individuals are different from monolinguals has been extensively studied, with a majority of researchers focusing on the better performance of bilinguals on tasks that measure certain cognitive abilities. Previous studies suggested that using two languages actively alters the executive functions, including inhibitory control and task shifting [5-11]. These cognitive abilities may be generalized to some non-linguistic domains. However, it remains unclear whether the bilingual advantages are still significant when we directly measure cognitive abilities using nonlinguistic tasks, and people are still looking for appropriate methods for studying it [19]. As a result, we want to solve the debate over whether a bilingual advantage exists in children by reviewing the current research addressing cognition in bilinguals from different cognitive perspectives, such as executive functions, linguistic abilities, and neurological changes.

\section{EVIDENCE FOR BILINGUAL ADVANTAGES}

\subsection{Executive Functions}

To begin with, being bilingual is essential and beneficial for young children's cognitive development, in particular executive functions. Executive functions (EFs) are "higher-order cognitive processes involved in goal-oriented behavior, such as planning and sequencing" [20]. In other words, EFs are adjustable actions that individuals are able to provide their own unique responses instead of having automatic and orthodox ideas and reactions [21]. These functions are significant and necessary for young children to solve original and novel academic and learning problems. The most often mentioned components of executive functions are inhibitory control, working memory and shifting [21]. Inhibition control is the capability of intentionally inhibiting impulsive responses, ideas and actions [20]. Working memory is the ability to conserve and manipulate information in the mind while adding or subtracting information [22]. Shifting also calls cognitive flexibility, meaning the ability to consciously disengage from one task for managing another task or goal [21]. Now, let's go through accurately about being bilingual is effective for children in each aspect of executive functions.

\subsubsection{Inhibitory Control}

Firstly, being bilingual could highly upgrade children's inhibitory control. Santillán \& Khurana claimed that being bilingual and immersing in bilingual environments are significantly aligned with quicker inhibitory control (IC) development [5]. They compared three groups of people differing in different language backgrounds, monolingual English children, Spanishbilingual children and children who transitioned from being Spanish monolingual to Spanish-English bilingual [5]. By undertaking a pencil-tap task which is a measure from Preschool Self-Regulation Assessment (PSRA) of IC, both children and tester have a pencil. Children should tap their pencil one time when the tester taps his or her pencil twice, or the tester taps it once and children tap it twice [5]. This task allows children to remember the task's rules in mind. As a result, SpanishEnglish bilinguals performed better than the other two groups. Hilchey \& Klein also suggested that young bilingual kids have better inhibitory control. By doing both compatible and incompatible trials, such as Simon tasks and Spatial troop, etc., bilingual children are more likely to suppress unrelated information comparing to monolinguals [6]. Moreover, Filippi et al. did research that tested whether young bilingual and monolingual children in the classroom can understand passive and active sentences in English [7]. The main task for children was to determine the target sentence with verbal interference. Children were instructed to neglect distractor sentences and pay attention to the target sentences [7]. The result displayed that bilingual children did better than monolingual children on this task due to bilinguals are more specific in apprehending complex sentences in the presence of linguistic interference [7].

Both Santillán \& Khurana and Hilchey \& Klein found common points that being bilingual is beneficial for inhibitory control. However, they still investigated different factors to influence the final result. For Santillán \& Khurana, they also inspected that the longer exposure of the second language is associated with 
enhanced development of IC [5]. According to Hilchey \& Klein, social economics status (SES) should be adequately controlled in studies. If researchers could highly control this factor, it might remove bilingual's advantages of inhibitory control [6].

\subsubsection{Working Memory}

Additionally, with the help of being bilingual, children also show enhancement in their working memory (WM). Daubert and Ramani suggested that young bilinguals' enhanced working memory could be a positive influencer for mathematical development [8]. They compared 74 bilinguals and monolinguals by completing both non-verbal WM tests and metathetical tests involving addition, numeral identification and nonsymbolic magnitude comparison tasks [8]. As a result, they found out that bilinguals outperform in both tests than monolinguals on working memory by controlling other factors, such as SES and age. It is an interesting point that they also concluded that bilinguals show higher scores on addition and numeral identifications tests [8]. Therefore, bilingual children may show their unique benefits on mathematical achievement and learning. Daubert \& Ramani stated that bilingual children with disruptive behavior disorders (DBDs) perform better than monolingual children on spatial working memory tasks [9]. They tested children working memory individually through both verbal and mathematical aspects by the Automated Working Memory Assessment (AWMA) [9]. What is more, they also discovered that bilinguals with DBDs exhibited fewer WM problems than monolinguals. Hence, being bilingual could also be an essential and protective factor contributing to children with DBDs [9]

\subsubsection{Shifting}

Thirdly, being bilingual for young children also has a positive impact on their shifting. Mehrani \& Zabihi declared that bilinguals not only show competence in linguistic aspects but also in executive functions, such as shifting and working memory [10]. They compared 36 Persian-Turkey young bilinguals and 31 Persian monolinguals by undertaking dimensional change card sort (DCCS), a test for measuring shifting ability. Children would sort cards with two dimensions of color and shape [10]. As a result, bilinguals showed higher scores on shifting tasks than monolinguals'. What is more, Prior \& Macwhinney also deserted that bilingual students displayed less switching costs than monolinguals by doing task-switching paradigm [11]. Meanwhile, they also discussed that lifelong bilingualism might essentially impact people's shifting abilities. In other words, bilinguals might garner improved proficiency in shifting between mental sets [11].

\subsection{Language Skills}

Besides the improvement in executive function skills, bilingualism's cognitive benefits can also be reflected in their enhanced language abilities. A substantial amount of studies have demonstrated that bilinguals, compared to their monolingual counterparts, are more easily able to acquire a third language [23]. One contributing factor to this superiority is the increased metalinguistic awareness [24]. Metalinguistic awareness is defined as the ability to reflect on language as an object and is closely tied to other aspects of language development, such as children's knowledge of words and concepts and their sentence comprehension [25]. One early study measured metalinguistic awareness among grade 3 and grade 6 bilingual children and the monolingual control group using language objectivity test, revealing that bilingual children in both grade groups showed a higher awareness of the arbitrary relationship between a word's meaning and its form [26]. Bialystok's review examined the metalinguistic development between bilingual and monolingual children and concluded that bilingualism was associated with a higher ability to reflect on the language and better performance on certain tasks such as those involving world awareness [22]. Moreover, previous studies have suggested an additive effect of bilingualism on L3 acquisition. In one study investigating the impact of bilingualism on vocabulary achievement in English among Iranian EFL learners, the bilingual group performed significantly better than the monolingual group, controlled for age, gender, and the level of instruction, on a controlled productive ability vocabulary test [23].

In addition to language learning ability that showed up later in life, previous research has also shown better visual language discrimination performances in bilingual infants comparing to monolingual infants. In the beginning stage of language acquisition, infants become attuned to the sounds and rules of their native language. A bilingual child is exposed to two languages at the same time and is able to keep them distinct. Weikum et al. showed silent video clips of talking faces to both monolingual and bilingual infants [24]. At eight months, the monolingual infants whose native language is English lost the ability to distinguish English from French. However, 8-month-old French-English bilingual infants can discriminate between the two languages.

\subsection{Neurological Changes}

Many studies have investigated the neurological differences between bilingual and monolingual individuals and found many structural and neuroplasticity differences. Actively using two languages drastically changed the everyday experiences of bilingual children, whose brains develop more rapidly than at any other time in life. Over time, being 
bilingual may rewire the brain and change the brain not only functionally but also neurologically. Many previous studies have shown the structural changes in people who have been actively using more than one language since childhood.

Klein et al.'s study compared the cortical thickness of simultaneous bilingual, sequential bilingual, and monolingual participants [14]. The MRI results showed that learning two languages sequentially had an effect on brain development. Specifically, there was a positive correlation between the age when a second language was acquired and the thickness of the left inferior frontal cortex. The left inferior frontal cortex is crucial for processing and generating speech; thus, the increased thickness in this area may explain bilingual's superior language learning ability compared to monolinguals [29]. Elmer et al.'s study also showed the induced neuroplasticity related to white matter architecture at the macrostructural level [30]. Concretely, comparing to the control group, professional simultaneous interpreters had a lower fractional anisotropy value in anterior and posterior parts of the corpus callosum, which is associated with reduced axial and enhanced radial diffusivity, suggesting a different and more complex structure with less parallel devolution. Similarly, Piliasikas et al.'s study found the structural change of white matter in bilingual individuals [31]. Using TractBased Spatial Statistics analysis, higher fractional anisotropy values were found in bilinguals compared to monolinguals in language-related WM tracts, which might give bilingual advantages on semantics and syntax processing.

\section{EVIDENCE AGAINST BILINGUAL ADVANTAGES}

Although previous research has found that bilingualism is beneficial to executive controls, language learning skills, and neurological functions, other studies have shown no such difference between monolinguals and bilinguals.

\subsection{Executive Functions}

In the study of 11,041 participants, Nichols et al. found no reliable differences in executive function between monolinguals and bilinguals [5]. Specifically, they used twelve cognitive tests to assess executive functions, such as working memory, problem-solving, and planning. The results demonstrated a bilingual benefit on Digit Span when groups were poorly matched; however, the effect is disappeared when the groups were matched in terms of country of birth, SES, and education. Thus, the researchers concluded that bilingualism does not have general cognitive advantages.

Further, Duñabeitia and colleagues tested the cognitive performance of bilingual and monolingual children to evaluate their inhibitory skills [29]. Specifically, they used a classical verbal version of the Stroop task and a nonverbal version of the same task. In the nonverbal version, they used the Attention Network Test (ANT) that requires participants to pay attention to an arrow and decide in which direction an arrow is pointing. This nonlinguistic task involved executive control as well as alerting and orienting networks. The results showed that both monolingual and bilingual children had equal performance in these two tasks. Also, the result was not affected by any independent factors investigated. Thus, even though much research supports bilingual inhibitory advantages, Duñabeitia et al. indicated that bilingual children do not have specific merits in inhibitory control compared to monolinguals. Additionally, Antón et al. also reached the same conclusion that there are no bilingual advantages in inhibitory skills [30], given that the performance of the ANT task of both monolingual and bilingual children was highly similar and indistinguishable.

\subsection{Metacognition}

Besides contradictory evidence of bilingual cognitive benefits, other studies focus on metacognition [6]. Metacognition is the ability to be aware of one's cognitive process [31], and it is usually evaluated by comparing participants' task performance and confidence judgements between correct and error trials [6]. For example, if participants are shown a series of pictures and are asked to evaluate whether they have seen them before, people with good metacognitive abilities would report a higher level of confidence when making correct judgments than when they were wrong. Specifically, Folke et al. used a two-alternative-forcedchoice task to assess metacognitive performance by comparing confidence judgments regarding accuracy, bias, and efficiency for bilinguals and monolinguals. Participants are asked to complete the digit span task, Raven's Matrices, and the Dot Discrimination task. The results found that bilingual participants were less confident in their judgement when they completed tasks correctly compared to monolinguals, indicating a bilingual disadvantage in metacognitive efficiency.

\section{CONCLUSION}

The aim of this paper was to investigate the debate of bilingual benefits in young children. On the one hand, we have demonstrated that bilingual children have better executive functions in three aspects compared to monolingual children. Specifically, bilingual children showed higher inhibitory controls by outperforming monolinguals in tasks requiring irrelevant information inhibition. They also exhibited the enhancement in their working memory, which has the potential benefit on mathematical learning. In addition, being bilingual for young children could result in improved shifting 
abilities in DCCS, indicating less switching cost for bilinguals to switch their attention between different mental sets. Further, we have discussed bilingual benefits associated with language skills. Bilingual children were easier to acquire a third language due to enhanced metalinguistic awareness than monolingual children. Bilingual infants also showed better visual language discrimination, helping them distinguish between two languages, while monolingual babies have lost the ability due to a lack of a second language. Lastly, we examined neurological differences between monolinguals and bilinguals and found that bilinguals have better neuroplasticity which promotes language learning regarding semantics and syntax processing.

On the other hand, we have reviewed the evidence that fails to detect a bilingual advantage in cognitive development. One study demonstrates that bilinguals and monolinguals had the same executive function performance. Subsequent studies examined the inhibitory skills and concluded that the inhibition difference between the two groups was too similar to distinguish. Thus, they reached the same conclusion that there is not a cognitive benefit associated with being bilingual. Moreover, bilinguals showed lower confidence in their judgments, demonstrating a bilingual disadvantage in metacognition. However, it should be cautious about interpreting null findings that didn't support the notion of bilingual cognitive benefits as they frequently contain methodological limitations. For instance, regarding the conclusion of Duñabeitia's [27] and Antón's [28] studies, Bak questioned whether these groups differed in living in rural versus urban areas and in accessing the same educational and other services [33]. He also pointed out that the generalizability was diminished when researchers replicated the null findings from the same sample across multiple studies. Further, although most authors spare no effort to defend the standards of their bilingual samples, they rarely paid attention to monolinguals.

Based on the articles we reviewed, clearly, there is more evidence supporting the cognitive benefits of being bilingual. However, we should be aware that studying bilinguals is notoriously problematic because it is difficult to control the various possible extraneous variables that may often play a role in group comparisons. Future studies should address the above concerns to create a more comprehensive study design. Taken together, in relation to the bilingual advantages in cognitive development, there may be possible confounding variables that have not been accounted for and tested, leading to ongoing debates. For example, SES is a potential factor that needs to be taken into consideration. It is well known that SES affects cognitive performance, and higher SES is associated with cognitive benefits [34]. However, much past research did not report SES information or control for unmatched SES between groups. Additionally, it is essential to manipulate the language background of bilinguals because the cognitive development of being bilingual may vary depending on the age at which the second language is acquired and the proficiency of each language [35]. Lastly, the age of participants also needs to be sifted through. Specifically, the advantage of being bilingual tends to be found more reliably in childhood or late adulthood, but it is elusive among young adults [36]. Our review mainly focused on young children; it is easier to find evidence supporting bilingual benefits than null results. However, since the impact of being bilingual on cognition varies across the life span, future studies should try to understand the developmental trajectory of some cognitive functions from different ages.

In a word, we examined the evidence on the benefits of bilingualism from both points of view and concluded that bilinguals have more advantages than disadvantages. Therefore, we suggest that future researchers consider the above-mentioned existing limitations and find a comprehensive direction for bilingual research.

\section{REFERENCES}

[1] Eurostat. (2019, April). Foreign language skills statistics. $\quad$ Retrieved from http://ec.europa.eu/eurostat/statisticsexplained/index.php/

Foreign_language_skills_statistics

[2] Statistics Canada. (2017, August). Census in brief: Linguistic diversity and multilingualism in Canadian homes. Retrieved from http://www12 .statcan.gc.ca/censusrecensement/2016/as-sa/98-200-x/2016010/98200-x2016010-eng.cfm

[3] United States Census Bureau. American Community Survey. Retrieved September 22, 2021, from

https://data.census.gov/cedsci/table?d=ACS $\% 205$ Year\%20Estimates\%20Data\%20Profiles\&tid=ACS DP5Y2018.DP02

[4] F. Grosjean, (2010). Bilingual: Life and reality. Cambridge, MA: Harvard University Press. http://dx.doi.org/10.4159/9780674056459

[5] J. Santillán, \& A. Khurana, (2018). Developmental associations between bilingual experience and inhibitory control trajectories in Head Start children. Developmental science, 21(4), e12624.

[6] M. D. Hilchey, \& R. M. Klein, (2011). Are there bilingual advantages on nonlinguistic interference tasks? Implications for the plasticity of executive control processes. Psychonomic bulletin \& review, $18(4), 625-658$. 
[7] R. Filippi, J. Morris, F. M. Richardson, P. Bright, M. S. Thomas, A. Karmiloff-Smith, \& V. Marian, (2015). Bilingual children show an advantage in controlling verbal interference during spoken language comprehension. Bilingualism: Language and Cognition, 18(3), 490-501.

[8] A. M. Garcia, R. Ros, K. C. Hart, \& P. A. Graziano, (2018). Comparing working memory in bilingual and monolingual Hispanic/Latino preschoolers with disruptive behavior disorders. Journal of experimental child psychology, 166, 535-548.

[9] E. N. Daubert, \& G. B. Ramani, (2019). Math and memory in bilingual preschoolers: The relations between bilingualism, working memory, and numerical knowledge. Journal of Cognition and Development, 20(3), 314-333.

[10] M. Melby-Lervåg, T. S. Redick, \& C. Hulme, (2016). Working Memory Training Does Not Improve Performance on Measures of Intelligence or Other Measures of "Far Transfer": Evidence From a Meta-Analytic Review. Perspectives on Psychological Science, 11(4), 512-534. https://doi.org/10.1177/1745691616635612

[11] J. Santillán, \& A. Khurana, (2018). Developmental associations between bilingual experience and inhibitory control trajectories in Head Start children. Developmental science, 21(4), e12624.

[12] Carmit Altman, Tamara Goldstein, and Sharon Armon-Lotem. "Vocabulary, metalinguistic awareness and language dominance among bilingual preschool children." Frontiers in Psychology 9 (2018): 1953.

[13] M. H. Keshavarz, \& H. Astaneh. "The impact of bilinguality on the learning of English vocabulary as a foreign language (L3)." International Journal of Bilingual Education and Bilingualism 7.4 (2004): 295-302.

[14] D. Klein, K. Mok, J. Chen \& K.E. Watkins, “Age of language learning shapes brain structure: a cortical thickness study of bilingual and monolingual individuals." Brain and language 131 (2014): 20-24

[15] E. S. Nichols, C. J. Wild, B., Stojanoski, M. E. Battista, \& A. M. Owen, (2020). Bilingualism affords no general cognitive advantages: A population study of executive function in 11,000 people. Psychological Science, 31(5), 548-567. https://doi.org/10.1177/0956797620903113

[16] J. A. Duñabeitia, J. A. Hernández, E. Antón, P. Macizo, A. Estévez, L. J. Fuentes, \& M. Carreiras, (2014). The inhibitory advantage in bilingual children revisited: Myth or reality? Experimental Psychology, 61(3), 234-251. https://doi.org/10.1027/1618-3169/a000243

[17] E. Antón, J. A. Duñabeitia, A. Estévez, J. A. Hernández, A. Castillo, L. J. Fuentes, D. J. Davidson, \& M. Carreiras, (2014). Is there a bilingual advantage in the ANT task? evidence from children. Frontiers in Psychology, 5, 398-398. https://doi.org/10.3389/fpsyg.2014.00398

[18] T. Folke, J. Ouzia, P. Bright, B. De Martino, \&R. Filippi, (2016). A bilingual disadvantage in metacognitive processing. Cognition, 150, 119-132. https://doi.org/10.1016/j.cognition.2016.02.008

[19] P. Baggetta, \& P. A. Alexander, (2016). Conceptualization and operationalization of executive function. Mind, Brain, and Education, $10(1), 10-33$

[20] P. Baggetta, \& P. A. Alexander, (2016). Conceptualization and operationalization of executive function. Mind, Brain, and Education, 10(1), 10-33.

[21] N. Garon, S. E. Bryson, \&I. M. Smith, (2008). Executive function in preschoolers: a review using an integrative framework. Psychological bulletin, 134(1), 31 .

[22] M. Melby-Lervåg, T. S. Redick, \& C. Hulme, (2016). Working Memory Training Does Not Improve Performance on Measures of Intelligence or Other Measures of "Far Transfer": Evidence From a Meta-Analytic Review. Perspectives on Psychological Science, 11(4), 512-534. https://doi.org/10.1177/1745691616635612

[23] J. Cenoz. "The influence of bilingualism on third language acquisition: Focus on multilingualism." Language teaching 46.1 (2013): 71-86.

[24] C. Altman, T. Goldstein, and S. Armon-Lotem. "Vocabulary, metalinguistic awareness and language dominance among bilingual preschool children." Frontiers in Psychology 9 (2018): 1953.

[25] C. L. Smith, \& H. Tager-Flusberg. Metalinguistic awareness and language development. Journal of Experimental Child Psychology. 1982 Dec 1;34(3):449-68.

[26] J. Cummins. "Bilingualism and the development of metalinguistic awareness." Journal of cross-cultural psychology 9.2 (1978): 131-149.

[27] E. Bialystok. "Metalinguistic aspects of bilingual processing." Annual review of applied linguistics 21 (2001): 169-181. 
[28] W. M. Weikum, A. Vouloumanos, J. Navarra, S. Soto-Faraco, N. Sebastian-Galles, J.F. Werker, "Visual language discrimination in infancy." Science 316.5828 (2007): 1159-1159.

[29] J. D. Gabrieli, R. A. Poldrack, \& J. E. Desmond, "The role of left prefrontal cortex in language and memory." Proceedings of the national Academy of Sciences 95.3 (1998): 906-913.

[30] S. Elmer, J. Hänggi, M. Meyer, \& L. Jänckeet, "Differential language expertise related to white matter architecture in regions subserving sensorymotor coupling, articulation, and interhemispheric transfer." Human brain mapping 32.12 (2011): 2064-2074.

[31] C. Pliatsikas, Elisavet Moschopoulou, and James Douglas Saddy. "The effects of bilingualism on the white matter structure of the brain." Proceedings of the National Academy of Sciences 112.5 (2015): 1334-1337.4.00443

[32] L. Williams, P. Parthasarathy, \& M. Molnar. (2021). Measures of Bilingual Cognition - From
Infancy to Adolescence. Journal of Cognition, 4(1), 45. DOI: http://doi.org/10.5334/joc. 184

[33] T. H. Bak (2016). The impact of bilingualism on cognitive ageing and dementia: Finding a path through a forest of confounding variables. Linguistic Approaches to Bilingualism, 6(1-2), 205-226. https://doi.org/10.1075/lab.15002.bak

[34] K. G. Noble, M. F. Norman, \& M. J. Farah (2005). Neurocognitive correlates of socioeconomic status in kindergarten children. Developmental Science, 8(1), 74-87. https://doi.org/10.1111/j.14677687.2005.00394.x

[35] L. Williams, P. Parthasarathy, \& M., Molnar (2021). Measures of bilingual cognition - from infancy to adolescence. Journal of Cognition, 4(1), 45-45.

[36] M. Antoniou (2019). The advantages of bilingualism debate. Annual Review of Linguistics, 5(1), 395-415. https://doi.org/10.1146/annurevlinguistics-011718-011820 